Center for

Mathematical Economics

Working Papers

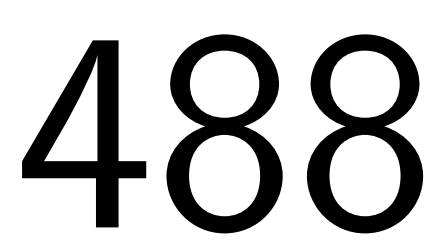

September 2013

\title{
Aggregation of Monotonic Bernoullian Archimedean preferences: Arrovian impossibility results
}

Frederik Herzberg

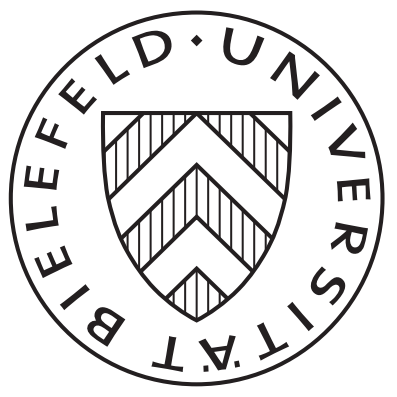




\title{
Aggregation of Monotonic Bernoullian Archimedean preferences: Arrovian impossibility results
}

\author{
Frederik Herzberg
}

\begin{abstract}
Cerreia-Vioglio, Ghirardato, Maccheroni, Marinacci and Siniscalchi (Economic Theory, 48:341-375, 2011) have recently proposed a very general axiomatisation of preferences in the presence of ambiguity, viz. Monotonic Bernoullian Archimedean (MBA) preference orderings. This paper investigates the problem of Arrovian aggregation of such preferences - and proves dictatorial impossibility results for both finite and infinite populations. Applications for the special case of aggregating expected-utility preferences are given. A novel proof methodology for special aggregation problems, based on model theory (in the sense of mathematical logic), is employed.

Key words: ambiguity; Knightian uncertainty; expected utility; Monotonic Bernoullian Archimedean (MBA) preferences; Arrovian social choice; Arrow's theorem; impossibility result; ultrafilter; ultraproduct

Journal of Economic Literature classification: D71, D81, C02

2010 Mathematics Subject Classification: 91B14, 91B06, 03C20, 03C 98
\end{abstract}

\section{Introduction}

The study of preferences in the presence of risks with unknown probability distribution (also known as second-order risk, Knightian uncertainty or ambiguity) has been a very active area of research in recent decades. Interest in axiomatising such preferences goes back at least to the seminal paper by Ellsberg [6]; since then, various authors have proposed axiomatisations of increasing generality, in particular Gilboa and Schmeidler [12], Föllmer and Schied [8, 9] as well as

Center for Mathematical Economics, Bielefeld University, Universitätsstraße 25, D-33615 Bielefeld, Germany.

Munich Center for Mathematical Philosophy, Ludwig Maximilian University of Munich, Geschwister-Scholl-Platz 1, D-80539 Munich, Germany. 
Maccheroni, Marinacci and Rustichini [23]. Recently, even gametheoretic aspects of ambiguity have been studied, for example by Riedel and Sass [24] and Kuzmics [19].

An even greater level of generality was recently achieved by CerreiaVioglio, Ghirardato, Maccheroni, Marinacci and Siniscalchi [4] with their proposal of axiomatising preferences in the presence of ambiguity as Monotonic Bernoullian Archimedean (MBA) preferences and their extensive study of the same. The MBA axiomatisation is distinguished by a comparatively low complexity, as measured by the number of quantifier alternations, which is the common complexity measure for formulae in mathematical logic.

The present paper is concerned with the question whether rational aggregation, in the sense of Arrovian social choice theory, of such preferences is possible. One consequence of the comparatively low complexity of the axiomatisation by Cerreia-Vioglio, Ghirardato, Maccheroni, Marinacci and Siniscalchi [4] is that said question can be answered relatively quickly and comprehensively using tools from model theory (a branch of mathematical logic): Based on the model-theoretic approach to abstract aggregation theory pioneered by Lauwers and Van Liedekerke [20] and more recently further developed by Herzberg and Eckert [16, 17], we prove that under some standard assumptions (a strong version of the independence axiom, the Pareto principle, and conditions ensuring the richness of the domain), nondictatorial Arrovian aggregation of MBA preferences is not possible, neither for finite nor (unlike in the case of allowing more general preferences, cf. Campbell [3]) infinite populations. While this proof methodology presently requires the set of states of the world to be finite, there is some hope that the use of infinitary model theory (cf. e.g. Keisler [18]) might eventually provide the means to overcome this limitation.

Of course, an obvious positive way to respond to Arrovian impossibility results, such as those contained in the present paper, is to drop the aggregator desideratum of independence of irrelevant alternatives or neutrality/systematicity. The resulting problem of mere Paretian aggregation is usually analytically more tractable. However, even Paretian aggregation is impossible for finite profiles of generalised expected-utility preferences, as discussed by several authors, including relatively recently Gajdos, Tallon and Vergnaud [10]. The main contribution of the present paper is the consideration of the case of infinite populations (featured, for example, in macroeconomics).

The structure of this paper is as follows. We review Montonic Bernoullian Archimedean preferences in Section 2, motivate the aggregation problem for MBA preferences in Section 3, formally state the formal results in Section 4 and conclude with a brief discussion in Section 5. The proofs of the main results are contained in Section 6 . 


\section{Monotonic Bernoullian Archimedean (MBA) preferences}

In the following, we briefly describe the axiomatisation of Monotonic Bernoullian Archimedean (MBA) preferences as well as the main representation theorem of Cerreia-Vioglio, Ghirardato, Maccheroni, Marinacci and Siniscalchi [4]. Herein, as was already pointed out in the introduction, we have to confine ourselves presently (for technical reasons) to the special case where there are only finitely many states of the world. The framework is along the lines of Anscombe and Aumann [1].

Let $S$ (the set of states of the world) be a finite set. Let $X$ (the set of consequences) be a convex subset, with at least two distinct elements, of an arbitrary vector space. An act is a function $f: S \rightarrow X$. The mixture of two acts is defined in the obvious way: For all $\alpha \in[0,1]$ and $f, g: S \rightarrow X$, the mixture $\alpha f+(1-\alpha) g$ is defined point-wise as $s \mapsto \alpha f(s)+(1-\alpha) g(s)$. (This is again an act as $X$ is convex.) Clearly, any element $x \in X$ can itself be viewed as an act, viz. the constant act $s \mapsto x$.

MBA preferences are special preference relations among acts:

DeFINITION 1. Consider a binary relation $\succsim$ on $X^{S}$ with symmetric part $\sim$ and asymmetric part $\succ$. The relation $\succsim$ is called a Monotonic, Bernoullian and Archimedean (MBA) preference relation if and only if it satisfies all of the following:

(1) $\succsim$ is non-trivial $]^{1}$ complete and transitive;

(2) for all acts $f, g$, if $f(s) \succsim g(s)$ for all $s \in S$, then $f \succsim g$ (Monotonicity Axiom);

(3) for all $x, y, z \in X$ and $\lambda \in(0,1]$, if $x \succ y$, then $\lambda x+(1-\lambda) z \succ$ $\lambda y+(1-\lambda) z$ (Bernoullian or Risk Independence Axiom);

(4) for all acts $f, g, h$, if $f \succ g \succ h$, then there exist $\alpha, \beta \in(0,1)$ such that $\alpha f+(1-\alpha) h \succ g \succ \beta f+(1-\beta) h$ (Continuity or Archimedean Axiom).

MBA preferences admit a beautiful representation theorem (Cerreia-Vioglio, Ghirardato, Maccheroni, Marinacci and Siniscalchi [4, Proposition 1]) that substantially generalises the well-known representation theorems for maxmin expected-utility preferences (cf. Gilboa and Schmeidler [12]) or variational preferences (cf. Maccheroni, Marinacci and Rustichini [23]). This representation theorem (and to some extent also its predecessors) requires the notion of monotonic normalised continuous functionals. Let $\Gamma$ be an interval $\subseteq \mathbf{R}$, and consider a functional $I: \Gamma^{S} \rightarrow \mathbf{R}$. We call $I$ monotonic if and only if $I(a) \leq I(b)$ whenever $a \leq b$ (point-wise on $S$ ); $I$ is called normalised if and only if $I$ maps the constant function $s \mapsto \alpha$ to $\alpha$ for all $\alpha \in \Gamma$. In light of the norm equivalence on finite-dimensional vector spaces, the

\footnotetext{
${ }^{1}$ In the sense that not for all acts $f, g, f \succsim g$.
} 
map $I$ is called continuous if it is continuous with respect to any norm on the finite-dimensional $\mathbf{R}^{S} \supseteq \Gamma^{S}$, e.g. the Euclidean norm.

TheOREM 2 (Cerreia-Vioglio et al.). A binary relation $\succsim$ on $X^{S}$ is an MBA preference relation if and only if there exist a non-constant affine function $u: X \rightarrow \mathbf{R}$ and a monotonic, normalised, continuous functional $I: u[X]^{S} \rightarrow \mathbf{R}$ such that for all $f, g: S \rightarrow X$,

$$
f \succsim g \Leftrightarrow I(u \circ f) \geq I(u \circ g) .
$$

The function $u$ and the functional I are unique up to affine monotone transformation.

An MBA preference relation $\succsim$ is an expected-utility preference relation if there exists some probability measure $p: 2^{S} \rightarrow[0,1]$ and some non-constant affine function $u: X \rightarrow \mathbf{R}$ such that for all $f, g: S \rightarrow X$,

$$
f \succsim g \Leftrightarrow \int u \circ f \mathrm{~d} p \geq \int u \circ g \mathrm{~d} p .
$$

Let henceforth $Q$ denote the set of all expected-utility preference relations.

\section{The question of Arrovian aggregation of MBA preferences}

Moving to the aggregate level, consider a population in which all individuals rank their available acts under ambiguity according to MBA preference relations. One may ask: Is it possible to merge ('aggregate') these preferences into a single MBA preference relation, in a way that respects certain responsiveness axioms? Put slightly differently: Is there a rational mechanism, other than dictatorship, by which a population of individuals holding MBA preferences can agree on a single aggregate MBA preference ordering? If the answer were affirmative, this would, for instance, entail a microeconomic foundation for the macroeconomic use of MBA preferences at the aggregate level, e.g. multiplier preferences (cf. Hansen and Sargent [13]) employed by a social planner.

If one employs responsiveness axioms that are derived from Arrovian social choice theory and restricts oneself to finite populations only, one may expect the answer to be negative, on account of Arrow's [2] impossibility theorem. For the case of infinite populations which has some potential interest for microfoundations in light of the continua-of-agents models used in macroeconomics - , one may hope that such an aggregation is feasible, on account of Fishburn's [7] possibility theorem.

As it turns out, however, the Archimedean axiom moves the problem towards the setting of Campbell's [3] impossibility theorem for aggregating continuous preference relations. Thus, there is a rather 
general result about Arrovian aggregation of MBA preferences — but, unfortunately, it is negative.

\section{Arrovian aggregation of MBA preferences is impossible}

In order to state the result, let us denote by $\mathcal{P}$ the set of MBA preferences, and let us fix an arbitrary non-empty set $N$, henceforth called the population. A typical element of $\mathcal{P}^{N}$ (profile) will be denoted by $\underline{P}=\left(P_{i}\right)_{i \in N}$. Whenever $P$ is a preference ordering, we write $P^{\succ}$ for its asymmetric part.

Let us introduce the following terminology.

- An aggregator of Montonic Bernoullian Archimedean preferences (for short: $M B A$ aggregator) is a map with domain $\subseteq \mathcal{P}^{N}$ and range $\subseteq \mathcal{P}$.

- An MBA aggregator $F$ is systematic if and only if for every $\underline{P}$ in the domain of $F$ and all $f, f^{\prime}, g, g^{\prime}: S \rightarrow X$,

$\left\{i \in N: f P_{i} g\right\}=\left\{i \in N: f^{\prime} P_{i} g^{\prime}\right\} \Rightarrow\left(f F(\underline{P}) g \Leftrightarrow f^{\prime} F(\underline{P}) g^{\prime}\right)$.

- An MBA aggregator $F$ is weakly Paretian if and only if for every $\underline{P}$ in the domain of $F$ and all $f, g: S \rightarrow X$, if $f P_{i} g$ for all $i \in N$, then $f F(\underline{P}) g$.

- An MBA aggregator $F$ is Paretian if and only if it is weakly Paretian and for every $\underline{P}$ in the domain of $F$ and all $f, g: S \rightarrow$ $X$, if $f P_{i}^{\succ} g$ for all $i \in N$, then $f F(\underline{P})^{\succ} g$.

- An MBA aggregator is dictatorial if and only if there exists some dictator $d \in N$ such that for every $\underline{P}$ in the domain of $F$ and all $f, g: S \rightarrow X$,

$$
f F(\underline{P}) g \Leftrightarrow f P_{d} g .
$$

- An MBA aggregator $F$ is universal if and only if its domain is $\mathcal{P}^{N}$.

- An MBA aggregator is weakly universal if and only if there are MBA preference relations $\succsim_{1}, \succsim_{2}, \succsim_{3}$ such that

(1) $f \succsim_{1} g, f^{\prime} \succsim_{1} g^{\prime}, f \succsim_{2} g, f^{\prime} \prec_{2} g^{\prime}, f \prec_{3} g, f^{\prime} \succsim_{3} g^{\prime}$ for some acts $f, f^{\prime}, g, g^{\prime}$, and

(2) the domain of $F$ contains $\left\{\succsim_{1}, \succsim_{2}, \succsim_{3}\right\}^{N}$.

- An MBA aggregator $F$ is said to have a large domain if and only if it is weakly universal, $N$ is infinite and there exists a profile $\left(P_{i}\right)_{i \in N}$ in its domain and some acts $f, g, h$ such that the set

$$
\left\{i \in N: f P_{i}^{\succ} g P_{i}^{\succ} h, \quad(\alpha f+(1-\alpha) h) P_{i}^{\succ} g P_{i}^{\succ}(\beta f+(1-\beta) h)\right\}
$$

is finite for all $\alpha, \beta \in(0,1)$ and such that $f F(\underline{P})^{\succ} g F(\underline{P})^{\succ} h$.

The concepts weak universality and large domain are not common and therefore require some explanation. Having a large domain means being defined for rather unusual profiles. For example, a Paretian 
aggregator has a large domain if its domain contains a profile of MBA preferences under which all individuals support the ranking $f \succ g \succ h$, but the "perturbed" ranking $\alpha f+(1-\alpha) f \succ g \succ \beta h+(1-\beta) h$ is always rejected by all but finitely many individuals - no matter how close $\alpha$ and $\beta$ are chosen to 1 and 0 , respectively. One might say that such a profile fails to be equicontinuous at the acts $f, g, h$ in a rather strong sense viz. that not even one of its (infinite) subsequences is equicontinuous.

For example, if $N$ is countably infinite, then all Paretian MBA aggregators whose domain comprises all profiles consisting of expectedutility preferences have a large domain:

REMARK 3. Suppose $S$ contains at least three distinct elements, let $N$ be countably infinite and $F$ be Paretian with domain $\supseteq Q^{N}$. Then $F$ has a large domain.

The notion of weak universality is well-chosen in the sense that universality implies weak universality:

REMARK 4. If $S$ contains at least two distinct elements, then any universal MBA aggregator is weakly universal.

Aggregators of expected-utility preferences are weakly universal:

REMARK 5. If $S$ contains at least two distinct elements, then any $M B A$ aggregator with domain $\supseteq Q^{N}$ is weakly universal.

The main finding of this paper is the following impossibility result:

Proposition 6. Let $F$ be a systematic and weakly Paretian aggregator of Montonic Bernoullian Archimedean preferences.

(1) If $N$ is finite and $F$ is weakly universal, then $F$ is dictatorial.

(2) If $N$ is infinite and $F$ has a large domain, then $F$ is dictatorial.

In particular, this entails that non-dictatorial Paretian systematic aggregation of expected-utility preferences is impossible, even for (countably) infinite populations.

Corollary 7. Suppose $S$ contains at least three distinct elements, and let $F$ be a systematic and weakly Paretian aggregator of Montonic Bernoullian Archimedean preferences whose domain contains at least all expected-utility preference profiles.

(1) If $N$ is finite, then $F$ is dictatorial.

(2) If $N$ is countably infinite and $F$ is even Paretian, then $F$ is dictatorial.

\section{Discussion}

The axiomatisation of preferences in the presence of ambiguity in terms of MBA preferences by Cerreia-Vioglio et al. [4] is remarkable 
on account of its great mathematical generality and elegant, simple logical form. The low degree of formal complexity permits the direct application of an aggregation-theoretic methodology that employs tools from mathematical logic. A relatively general impossibility theorem, encompassing both finite and infinite electorates, follows.

Hence, the aggregation of MBA preferences cannot follow Arrovian lines, not even on infinite populations. New avenues may open if one significantly weakens or jettisons independence-like conditions (such as the strong independence notion of systematicity - also known as neutrality - in this paper): For the very special case of von Neumann-Morgenstern preferences, it has long been known that mere Paretian aggregation is possible even for finite electorates (cf. Harsanyi [14]) while Arrovian aggregation is not (cf. Le Breton [21]). The Paretian aggregation of a large class of preference relations, including the Gilboa-Schmeidler maxmin expected-utility preferences, has been investigated by Gilboa, Schmeidler and Samet [11, Chambers and Hayashi [5] as well as Gajdos, Tallon and Vergnaud [10]. For the case of finite electorates, their impossibility result is similar to ours and even stronger as it dispenses of any independence or systematicity conditions; however, they do not treat the infinite case, which is the most important contribution of the present paper.

In any case, the cost thus abandoning an Arrovian approach to aggregation theory is, as in the Harsanyi-inspired literature, is of course that one leaves behind the most firmly established framework of social choice theory. For example, one may very well question whether a mere Paretian aggregate of MBA preference orderings should be seen as a compelling microeconomic foundation of a macroeconomic use of MBA preference relations at the aggregate level. In view of the impossibility results of this paper, it appears unlikely that MBA preferences at the aggregate level can be given a general microeconomic justification.

Analogous results have been obtained with similar methods for the special case of variational preferences (cf. Herzberg [15]). However, due to the simple and elegant axiomatisation of MBA preferences, the proof of the impossibility result in this paper is much more appealing.

\section{Proofs}

Proof of Remark [3, Let $N$ be countably infinite. Without loss of generality, we may assume that $N$ equals the set $\mathbf{N}$ of nonnegative integers. Let $s_{0}, s_{1}, s_{2}$ be three distinct elements of $S$ and $F$ Paretian with domain $\supseteq 2^{\mathbf{N}}$.

Let $x_{0}, x_{1}$ be two distinct elements of $X$. Let $u: X \rightarrow \mathbf{R}$ be a non-constant and affine function satisfying $u\left(x_{1}\right)=1$ and $u\left(x_{0}\right)=0$. Let, for each $k \in\{0,1,2\}, f_{k}: S \rightarrow X$ be such that $f_{k}(s)=x_{1}$ if $s=s_{k}$ and $f_{k}(s)=x_{0}$ for $s \in S \backslash\left\{s_{k}\right\}$, so that $u \circ f_{k}(s)=1$ if $s=s_{k}$ and 
$u \circ f_{k}(s)=0$ for $s \in S \backslash\left\{s_{k}\right\}$. Define, for all $i \in \mathbf{N}$,

$$
a_{i}:=\int_{S} u \circ f_{0} \mathrm{~d} p_{i}, \quad b_{i}:=\int_{S} u \circ f_{1} \mathrm{~d} p_{i}, \quad c_{i}:=\int_{S} u \circ f_{2} \mathrm{~d} p_{i} .
$$

Then, clearly,

$$
a_{i}=p_{i}\left\{s_{0}\right\}, \quad b_{i}=p_{i}\left\{s_{1}\right\}, \quad c_{i}=p_{i}\left\{s_{2}\right\} .
$$

Let, for each $i \in \mathbf{N}, p_{i}$ be chosen to be concentrated on $\left\{s_{0}, s_{1}, s_{2}\right\}$ and such that

$$
a_{i}=p_{i}\left\{s_{0}\right\}=b_{i}+4^{-i-2}, \quad c_{i}=p_{i}\left\{s_{2}\right\}=b_{i}-2^{-i-2},
$$

whence

$$
b_{i}=p_{i}\left\{s_{1}\right\}=\frac{1}{3}\left(1-4^{-i-2}+2^{-i-2}\right) .
$$

Then for all $\alpha \in(0,1)$, one has the following chain of equivalences:

$$
\begin{aligned}
& \alpha a_{i}+(1-\alpha) c_{i}>b_{i} \Leftrightarrow \alpha\left(a_{i}-b_{i}\right)+(1-\alpha)\left(c_{i}-b_{i}\right)>0 \\
\Leftrightarrow & 4^{-i-2} \alpha-2^{-i-2}(1-\alpha)>0 \Leftrightarrow \alpha-2^{i+2}(1-\alpha)>0 \\
\Leftrightarrow & \alpha\left(1+2^{i+2}\right)>2^{i+2} \Leftrightarrow \frac{2^{i+2}}{1+2^{i+2}}<\alpha,
\end{aligned}
$$

and the last inequality can only hold for finitely many $i \in \mathbf{N}$ if $\alpha \in(0,1)$ since $\frac{2^{i+2}}{1+2^{i+2}} \longrightarrow 1$ as $i \rightarrow \infty$. Hence, the inequality

$$
\alpha a_{i}+(1-\alpha) c_{i}>b_{i}
$$

is only satisfied for finitely many $i \in \mathbf{N}$. Moreover, clearly

$$
a_{i}>b_{i}>c_{i}
$$

for every $i \in \mathbf{N}$.

Now let $\underline{P} \in Q^{\mathbf{N}}$ be the unique profile which satisfies for all $i \in \mathbf{N}$ and all $h, \tilde{h}: S \rightarrow X$,

$$
h P_{i} \tilde{h} \Leftrightarrow \int_{S} u \circ h \mathrm{~d} p_{i} \geq \int_{S} u \circ \tilde{h} \mathrm{~d} p_{i},
$$

and put

$$
f:=f_{0}, \quad g:=f_{1}, \quad h:=f_{2} .
$$

Then, by our construction,

$$
f P_{i}^{\succ} g P_{i}^{\succ} h
$$

(as $a_{i}>b_{i}>c_{i}$ ) for all $i \in \mathbf{N}$. Since $F$ is Paretian, this implies

$$
f F(\underline{P})^{\succ} g F(\underline{P})^{\succ} h \text {. }
$$

Moreover, the individual preference ranking

$$
(\alpha f+(1-\alpha) h) P_{i}^{\succ} g
$$

(which is equivalent to $\alpha a_{i}+(1-\alpha) c_{i}>b_{i}$ ) holds only for finitely many $i \in \mathbf{N}$ whenever $\alpha \in(0,1)$. Therefore, with $\underline{P}$ and $f, g, h$ as previously defined, the conditions in the definition of a large domain are satisfied. 
Proof of REMARK 4. It is enough to construct $\succsim_{1}, \succsim_{2}, \succsim_{3} \in \mathcal{P}$ and $f, g: S \rightarrow X$ such that $f \sim_{1} g, f \succ_{2} g$ and $f \prec_{3} g$. Then one can simply put $f^{\prime}:=g$ and $g^{\prime}:=f$ and $\mathcal{P}^{N}$ satisfies the requirements on the domain of a weakly universal aggregator.

The construction of such $\succsim_{1}, \succsim_{2}, \succsim_{3} \in \mathcal{P}$ and $f, g: S \rightarrow X$ is straightforward, thanks to the representation theorem of CerreiaVioglio et al. [4, Propositions 1] (Theorem 2).

Let $x_{0}, x_{1} \in X$ be two distinct outcomes, and let $u: X \rightarrow \mathbf{R}$ be a non-constant affine function; without losing generality, $u\left(x_{0}\right)<u\left(x_{1}\right)$. Let $s_{0}, s_{1} \in S$ be distinct, too, and choose $f, g$ such that $f\left(s_{0}\right)=x_{0}$, $f\left(s_{1}\right)=x_{1}, g\left(s_{0}\right)=x_{1}, g\left(s_{1}\right)=x_{0}$. Let $\Delta\left(s_{0}, s_{1}\right)$ be the set of all probability measures on $S$ that are actually concentrated on $\left\{s_{0}, s_{1}\right\}$, and let us define $I_{1}, I_{2}, I_{3}$ as follows:

$$
\begin{array}{rlrl}
I_{1}: & a \mapsto \min _{p \in \Delta\left(s_{0}, s_{1}\right)} \int a \mathrm{~d} p \\
I_{2}: & & a \mapsto u\left(x_{1}\right)+\min _{p \in \Delta\left(s_{0}, s_{1}\right)} \int(a-u \circ f) \mathrm{d} p \\
I_{3}: & & a \mapsto u\left(x_{1}\right)+\min _{p \in \Delta\left(s_{0}, s_{1}\right)} \int(a-u \circ g) \mathrm{d} p
\end{array}
$$

Now, clearly for all $h: S \rightarrow X$,

$$
\begin{aligned}
\min _{p \in \Delta\left(s_{0}, s_{1}\right)} \int u \circ h \mathrm{~d} p & =\min _{q \in[0,1]}\left(q u \circ h\left(s_{0}\right)+(1-q) u \circ h\left(s_{1}\right)\right) \\
& =u \circ h\left(s_{1}\right)+\min _{q \in[0,1]} q\left(u \circ h\left(s_{0}\right)-u \circ h\left(s_{1}\right)\right) \\
\min _{p \in \Delta\left(s_{0}, s_{1}\right)} \int-u \circ h \mathrm{~d} p & =\min _{q \in[0,1]}\left(-q u \circ h\left(s_{0}\right)-(1-q) u \circ h\left(s_{1}\right)\right) \\
& =-u \circ h\left(s_{1}\right)+\min _{q \in[0,1]} q\left(u \circ h\left(s_{1}\right)-u \circ h\left(s_{0}\right)\right),
\end{aligned}
$$

which can be used to verify that $I_{2}, I_{3}$ are normalised (that $I_{1}$ is normalised and that all of them are continuous and monotonic is obvious), and in a similar vein for all $h, \tilde{h}: S \rightarrow X$,

$$
\begin{aligned}
& \min _{p \in \Delta\left(s_{0}, s_{1}\right)}\left(\int u \circ h \mathrm{~d} p-\int u \circ \tilde{h} \mathrm{~d} p\right) \\
= & \min _{q \in[0,1]}\left(q u \circ h\left(s_{0}\right)+(1-q) u \circ h\left(s_{1}\right)-q u \circ \tilde{h}\left(s_{0}\right)-(1-q) u \circ \tilde{h}\left(s_{1}\right)\right) \\
= & u \circ h\left(s_{1}\right)-u \circ \tilde{h}\left(s_{1}\right) \\
& +\min _{q \in[0,1]} q\left(u \circ h\left(s_{0}\right)-u \circ \tilde{h}\left(s_{0}\right)-u \circ h\left(s_{1}\right)+u \circ \tilde{h}\left(s_{1}\right)\right)
\end{aligned}
$$


From here, we can deduce for our above choices of $f, g$, exploiting $u\left(x_{0}\right)<u\left(x_{1}\right)$ :

$$
\begin{aligned}
I_{1}(u \circ f) & =u\left(x_{1}\right)+u\left(x_{0}\right)-u\left(x_{1}\right)=u\left(x_{0}\right) \\
I_{1}(u \circ g) & =u\left(x_{0}\right)+0=u\left(x_{0}\right) \\
I_{2}(u \circ f) & =u\left(x_{1}\right)+0=u\left(x_{1}\right) \\
I_{2}(u \circ g) & =u\left(x_{1}\right)+u\left(x_{0}\right)-u\left(x_{1}\right)+0=u\left(x_{0}\right) \\
I_{3}(u \circ f) & =u\left(x_{1}\right)+u\left(x_{1}\right)-u\left(x_{0}\right)+2\left(u\left(x_{0}\right)-u\left(x_{1}\right)\right)=u\left(x_{0}\right) \\
I_{3}(u \circ g) & =u\left(x_{1}\right)+0=u\left(x_{1}\right)
\end{aligned}
$$

Thus,

$$
I_{1}(u \circ f)=I_{1}(u \circ g), \quad I_{2}(u \circ f)>I_{2}(u \circ g), \quad I_{3}(u \circ f)<I_{3}(u \circ g)
$$

and therefore

$$
f \sim_{1} g, \quad f \succ_{2} g, \quad f \prec_{3} g
$$

if $\succsim_{1}, \succsim_{2}, \succsim_{3}$ are the preference relations represented by $I_{1} \circ u, I_{2} \circ u, I_{3} \circ$ $u$, respectively.

Proof of Remark 5, As in the proof of Remark 4, it is sufficient to construct expected-utility preferences $\succsim_{1}, \succsim_{2}, \succsim_{3}$ and $f, g: S \rightarrow X$ such that $f \sim_{1} g, f \succ_{2} g$ and $f \prec_{3} g$. Then one can simply put $f^{\prime}:=g$ and $g^{\prime}:=f$ and $\mathcal{P}^{N}$ satisfies the requirements on the domain of a weakly universal aggregator.

And again, the construction of such $\succsim_{1}, \succsim_{2}, \succsim_{3} \in \mathcal{P}$ and $f, g: S \rightarrow X$ is straightforward, thanks to the representation theorem of CerreiaVioglio et al. [4, Propositions 1] (Theorem 2).

Let $x_{0}, x_{1} \in X$ be two distinct outcomes, and let $u: X \rightarrow \mathbf{R}$ be a non-constant affine function; without losing generality, $u\left(x_{0}\right)<u\left(x_{1}\right)$. Let $s_{0}, s_{1} \in S$ be distinct, too, and choose $f, g$ such that $f\left(s_{0}\right)=x_{0}$, $f\left(s_{1}\right)=x_{1}, g\left(s_{0}\right)=x_{1}, g\left(s_{1}\right)=x_{0}$. Let, for any $s \in S, \delta_{s}$ denote the Dirac measure concentrated on $s$ (i.e. for all $A \in 2^{S}, \delta_{s}(A)=1$ if $s \in A$ and $=0$ else). Let us define $I_{1}, I_{2}, I_{3}$ as follows:

$$
\begin{aligned}
I_{1}: & a \mapsto \int a \mathrm{~d} \frac{\delta_{s_{0}}+\delta_{s_{1}}}{2}=\frac{a\left(s_{0}\right)+a\left(s_{1}\right)}{2} \\
I_{2}: & a \mapsto \int a \mathrm{~d} \delta_{s_{1}}=a\left(s_{1}\right) \\
I_{3}: & a \mapsto \int a \mathrm{~d} \delta_{s_{0}}=a\left(s_{0}\right)
\end{aligned}
$$


Obviously then, $I_{1}, I_{2}, I_{3}$ are monotonic, normalised and continuous. Moreover, by our above choices of $f, g$, we obtain:

$$
\begin{array}{rlrl}
I_{1}(u \circ f)=\frac{u\left(x_{0}\right)+u\left(x_{1}\right)}{2}, & & I_{1}(u \circ g)=\frac{u\left(x_{1}\right)+u\left(x_{0}\right)}{2} \\
I_{2}(u \circ f)=u\left(x_{1}\right), & I_{2}(u \circ g)=u\left(x_{0}\right) \\
I_{3}(u \circ f)=u\left(x_{0}\right), & I_{3}(u \circ g)=u\left(x_{1}\right)
\end{array}
$$

Thus, exploiting our choice of $u$ (viz. $u\left(x_{0}\right)<u\left(x_{1}\right)$ ),

$$
I_{1}(u \circ f)=I_{1}(u \circ g), \quad I_{2}(u \circ f)>I_{2}(u \circ g), \quad I_{3}(u \circ f)<I_{3}(u \circ g)
$$

and therefore

$$
f \sim_{1} g, \quad f \succ_{2} g, \quad f \prec_{3} g
$$

if $\succsim_{1}, \succsim_{2}, \succsim_{3}$ are the preference relations represented by $I_{1} \circ u, I_{2} \circ u, I_{3} \circ$ $u$, respectively.

Proof of Proposition 6. For the proof of the main result, a purely technical reformulation is required. The model-theoretic approach to aggregation theory assumes that all relations involved are defined on the same set, in our case the set of acts. This means, in particular, that we need to find a way of viewing scalars weights as acts and hence the mixture operator as a ternary operation on acts.

In order to achieve this, fix two distinct elements of $x_{0}, x_{1} \in X$. For each $\alpha \in[0,1]$, we identify $\alpha$ with the constant act $\alpha x_{0}+(1-\alpha) x_{1}$. It is easy to verify that $\alpha x_{0}+(1-\alpha) x_{1}$ is invertible. Therefore, the mixture operator can be viewed as mapping a triple - consisting of a constant act of the form $\alpha x_{0}+(1-\alpha) x_{1}$ and two other acts - to another act, viz. the one defined by $\alpha f+(1-\alpha) g$. In addition, the definition of an MBA preference relation invokes the notion of a constant act and of the evaluation of an act at a certain state of the world $s$.

In this vein, all the axioms describing an MBA preference relation can be formulated using

- the strict preference relation symbol (denoted by $R$ in the following),

- the predicate of being a constant act (denoted by $C$ ),

- the predicate of being an act of the form $\alpha x_{0}+(1-\alpha) x_{1}$ (denoted by $I$ ),

- for each state of the world $s$, the formal projection operator $\pi_{s}$ that assigns to any act $f$ the constant act $f(s)$,

- the formal mixture operator $m$, whose interpretation is that to any triple consisting of two acts $f, g$ and an act of the form $\alpha x_{0}+(1-\alpha) x_{1}$ assigns $\alpha f+(1-\alpha) g$.

and the usual logical connectives and quantifiers of first-order predicate logic.

For purely technical reasons we need to find a formalisation of the Archimedean axiom which does not contain negations; therefore, 
the strict preference relation symbol has to be used as a fundamental symbol. Since the preference orderings are assumed to be complete, we can recover the weak preference relation $P$ and the symmetric part $Q$ of $P$ from $R$ by introducing

$$
f P g: \equiv \neg g R p, \quad f Q g: \equiv f P g \wedge g P f .
$$

In this formalisation, the MBA preference axioms are either universal, (i.e. of the form $(\forall f)(\forall g)(\forall h) \varphi$ where $\varphi$ is quantifier-free) or - in the case of the Archimedean axioms - universal-existential (i.e. of the form $(\forall f)(\forall g)(\forall h)\left(\exists f^{\prime}\right)\left(\exists g^{\prime}\right) \phi$ where $\phi$ is quantifier-free. Of course, any preference ordering $P$ among acts gives rise to a first-order structure - in the sense of model theory - whose domain is the set of acts and which interprets $R, C, I,(\pi(s))_{s \in S}, m$.

Using an extension, due to Herzberg and Eckert [17, of the model-theoretic approach to aggregation theory originally pioneered by Lauwers and Van Liedekerke [20], one can now prove that neither for finite nor for infinite electorates, there can be any non-dictatorial aggregators satisfying the conditions of the theorem. At the heart of the proof in both cases are the following two crucial facts. First, every weakly universal systematic weakly Paretian aggregator maps any profile $\underline{P}$ in its domain to the restriction, to the set of acts, of the ultraproduct of (the sequence of first-order structures obtained from) $\underline{P}$ with respect to some ultrafilter $\mathcal{D}_{F}$ [17, Lemma 3.5, Lemma 3.8]. Secondly, if an ultrafilter contains a finite set, it must be principal, i.e. the system of supersets of some singleton - in other words, it must be of the form $\{C \subseteq N: d \in C\}$ for some $d \in N$. Therefore, if an ultrafilter contains a finite set, one can infer that $F$ is a dictatorship: In order to verify that $d$ is the dictator ruling under $F$, it is enough to note that $F$ is the ultraproduct construction with respect to $\mathcal{D}_{F}$ restricted to the set of acts (Herzberg and Eckert [17, Lemma 3.8]), whence it follows that actually

$$
F\left(\underline{P}^{\prime}\right)=P_{d}^{\prime}
$$

for all profiles $\underline{P}^{\prime}$ in its domain.

Hence, in order to prove the dictatorial nature of $F$, it is sufficient to verify that indeed there is a finite subset of $N$ among the elements of the ultrafilter $\mathcal{D}_{F}$. In the case of a finite electorate, this is trivial, because $N \in \mathcal{D}_{F}$ by the definition of an ultrafilter and $N$ is finite by assumption.

In the case of an infinite electorate, more work and an additional assumption in the guise of the above large domain condition is needed. Let us first note that in the above logical formalisation, the Archimedean Axiom reads

$$
\forall f \forall g \forall h \exists a \exists b\left(I a \wedge I b \wedge\left(\begin{array}{c}
(f R g \wedge g R h) \rightarrow \\
(m(a, f, h) R g \wedge g R m(b, f, h))
\end{array}\right)\right)
$$


Let us now fix acts $f, g, h$ and a profile $\underline{P}$ as in the large domain condition and consider the formula

$$
\phi[a, b]: \equiv I a \wedge I b \wedge f R g \wedge g R h \wedge m(a, f, h) R g \wedge g R m(b, f, h),
$$

which has the - from a model-theoretic vantage point rather nice - property that it is free of universal quantifiers, negations and disjunctions, which we shall exploit shortly.

If $\succsim$ is an arbitrary preference ordering, we shall write $\succsim \models \phi[a, b]$ (read: $\phi[a, b]$ holds for $\succsim$ ) if and only if it holds when the asymmetric part $\succ$ of $\succsim$ is inserted for $R$ in $\phi[a, b]$, in other words, if and only if indeed $a, b$ are acts of the form $a=\alpha x_{0}+(1-\alpha) x_{1}, \quad b=\beta x_{0}+(1-\beta) x_{1}$ for $\alpha, \beta \in(0,1)$ and

$$
f \succ g \succ h, \quad \alpha f+(1-\alpha) h \succ g \succ \beta f+(1-\beta) h .
$$

Observe that for any preference ordering $P$ that satisfies the Archimedean Axiom as well as $f P^{\succ} g P^{\succ} h$, we have the existence of some $\alpha, \beta \in(0,1)$ such that

$$
\alpha f+(1-\alpha) h \succ g \succ \beta f+(1-\beta) h .
$$

In particular, in our new notation, this means that

(1) for all $P \in \mathcal{P}$, if $f P^{\succ} g P^{\succ} h$, then for some $a, b, P \models \phi[a, b]$.

By Herzberg and Eckert [17, Lemma 3.8], the aggregate $F(\underline{P})$ of any profile $\underline{P}=\left(P_{i}\right)_{i \in N}$ in the domain of $F$ can be seen as the restriction of the ultraproduct of $\underline{P}$ - more precisely: of the sequence of formal structures corresponding to the $P_{i}-$ to $X^{S}$ with respect to some ultrafilter $\mathcal{D}_{F}$ on $N$.

Combining this model-theoretic description of $F(\underline{P})$ with the fact that $\phi[a, b]$ is free of universal quantifiers, negations and disjunctions, the inductive proof (in formula complexity) of the fundamental theorem on ultraproducts (Łoś's theorem [22]) shows: for all $a, b, F(\underline{P}) \models \phi[a, b]$ if and only if the collection of those $i$ with $P_{i} \models \phi[a, b]$ is an element of the ultrafilter $\mathcal{D}_{F}$. Now, by our observation (1), there must be acts $a, b$ such that $F(\underline{P}) \models \phi[a, b]$, because (i) $F(\underline{P}) \in \mathcal{P}$ (F being an MBA aggregator) and (ii) our choice of $f, g, h, \underline{P}$ ensures that $f F(\underline{P})^{\succ} g F(\underline{P})^{\succ} h$. It follows that indeed $\left\{i \in N: P_{i}=\phi[a, b]\right\} \in \mathcal{D}_{F}$ for some acts $a, b$.

On the other hand, by our choice of $f, g, h$ and $\underline{P}$ as in the large domain condition, the coalition $\left\{i \in N: P_{i}=\phi[a, b]\right\}$ is actually finite. Hence, we have shown that among the elements of $\mathcal{D}_{F}$ there is indeed a finite subset of $N$. As was explained before (when proving the result for the finite case), this entails that $\left(\mathcal{D}_{F}\right.$ is principal and hence) $F$ is a dictatorship. 


\section{References}

[1] F.J. Anscombe and R.J. Aumann. A definition of subjective probability. Annals of Mathematical Statistics, 34:199-205, 1963.

[2] K.J. Arrow. Social choice and individual values, volume 12 of Cowles Commission Monographs. Wiley, New York, 2 edition, 1963.

[3] D.E. Campbell. Intergenerational social choice without the Pareto principle. Journal of Economic Theory, 50(2):414-423, 1990.

[4] S. Cerreia-Vioglio, P. Ghirardato, F. Maccheroni, M. Marinacci, and M. Siniscalchi. Rational preferences under ambiguity. Economic Theory, 48(23):341-375, 2011.

[5] C.P. Chambers and T. Hayashi. Preference aggregation under uncertainty: Savage vs. Pareto. Games and Economic Behavior, 54(2):430-440, 2006.

[6] D. Ellsberg. Risk, ambiguity, and the Savage axioms. Quarterly Journal of Economics, 75(4):643-669, 1961.

[7] P.C. Fishburn. Arrow's impossibility theorem: concise proof and infinite voters. Journal of Economic Theory, 2(1):103-106, 1970.

[8] H. Föllmer and A. Schied. Convex measures of risk and trading constraints. Finance and Stochastics, 6(4):429-447, 2002.

[9] H. Föllmer and A. Schied. Stochastic finance. An introduction in discrete time, volume 27 of de Gruyter Studies in Mathematics. de Gruyter, Berlin, 2nd edition, 2004.

[10] T. Gajdos, J.M. Tallon, and J.C. Vergnaud. Representation and aggregation of preferences under uncertainty. Journal of Economic Theory, 141(1):68-99, 2008.

[11] I. Gilboa, D. Samet, and D. Schmeidler. Utilitarian aggregation of beliefs and tastes. Journal of Political Economy, 112(4):932-938, 2004.

[12] I. Gilboa and D. Schmeidler. Maxmin expected utility with non-unique prior. Journal of Mathematical Economics, 18(2):141-153, 1989.

[13] L.P. Hansen and T.J. Sargent. Robust control and model uncertainty. American Economic Review, 91(2):60-66, 2001.

[14] J.C. Harsanyi. Cardinal welfare, individualistic ethics, and interpersonal comparisons of utility. Journal of Political Economy, 63(4):309-321, 1955.

[15] F.S. Herzberg. The (im)possibility of collective risk measurement: Arrovian aggregation of variational preferences. Economic Theory Bulletin, 1(1):69-92, 2013.

[16] F.S Herzberg and D. Eckert. Impossibility results for infinite-electorate abstract aggregation rules. Journal of Philosophical Logic, 41(1):273-286, 2012.

[17] F.S. Herzberg and D. Eckert. The model-theoretic approach to aggregation: Impossibility results for finite and infinite electorates. Mathematical Social Sciences, 64(1):41-47, 2012.

[18] H.J. Keisler. Model theory for infinitary logic. Logic with countable conjunctions and finite quantifiers, volume 62 of Studies in Logic and the Foundations of Mathematics. North-Holland, Amsterdam, 1971.

[19] C. Kuzmics. A rational ambiguity averse person will never display her ambiguity aversion. Technical report, Center for Mathematical Economics, Bielefeld University, April 2013.

[20] L. Lauwers and L. Van Liedekerke. Ultraproducts and aggregation. Journal of Mathematical Economics, 24(3):217-237, 1995.

[21] M. Le Breton. Essais sur les fondements de l'analyse économique de l'inégalité. Thèse pour le Doctorat d'État en Sciences Économiques, Université de Rennes 1, 1986. 
IMPOSSIBILITY OF ARROVIAN AGGREGATION OF MBA PREFERENCES 15

[22] J. Łoś. Quelques remarques, théorèmes et problèmes sur les classes définissables d'algèbres. In Th. Skolem, G. Hasenjaeger, G. Kreisel, A. Robinson, H. Wang, L. Henkin, and J. Łoś, editors, Mathematical interpretation of formal systems, volume 16 of Studies in Logic and the Foundations of Mathematics, pages 98113. North-Holland, Amsterdam, 1955.

[23] F. Maccheroni, M. Marinacci, and A. Rustichini. Ambiguity aversion, robustness, and the variational representation of preferences. Econometrica, 74(6):1447-1498, 2006.

[24] F. Riedel and L. Sass. Ellsberg games. Theory and Decision, forthcoming, 2013. 